\title{
Rapid and Simultaneous Determination of Ginsenosides Rb1, Rb2, Rc and Re in Korean Red Ginseng Extract by HPLC using Mass/Mass Spectrometry and UV Detection
}

\author{
Young-Min Kwon, Sung-Dong Lee, Hyun-Sook Kang, Mu-Gung Cho, Soon-Sun Hong*, Chae-Kyu Park**, \\ Jong-Tae Lee**, Byeong-Seon Jeon**, Sung-Ryong Ko**, Hyun-Joo Shon** and Dal-Woong Choi ${ }^{*}$ \\ College of Health Science, Korea University, Seoul 136-703, Korea \\ *College of Medicine, Inha University, Inchon 402-751, Korea \\ **KT\&G Central Research Institute, Daejeon 305-805, Korea \\ (Received December 16, 2008; Accepted December 22, 2008)
}

\begin{abstract}
For evaluating the quality of ginseng, simple and fast analysis methods are needed to determine the ginsenoside content of the ginseng products. The aim of this study was therefore to optimize conditions for fast analysis of the ginsenosides, the active ingredients in extracts of Korean red ginseng. When tandem HPLC mass spectrometry (HPLC-MS/ MS) was used, four forms of ginsenoside, $\mathrm{Rb} 1, \mathrm{Rb} 2, \mathrm{Rc}$, and $\mathrm{Re}$, were readily separated in seven minutes using a gradient mobile phase (acetonitrile and water containing acetic acid). This is the shortest separation time reported among the studies of major ginsenoside analysis. When gradient HPLC with UV detection was used, the detection limit was high, but separation of these four ginsenosides required 25 minutes using acetonitrile and water containing formic acid as a mobile phase. HPLC-MS/MS was able to separate ginsenoside Rg1 easily regardless of the mobile phase condition, but the HPLC-UV could not separate Rg1 because acetonitrile concentration in the mobile phase had to be maintained below $20 \%$. Ginsenoside peaks were clearer and had more sensitive detection limits when Korean red ginseng extract was analyzed by the HPLC-MS/MS, but the UV detection was useful for chromatographic fingerprinting of all four major ginsenosides of the extract: Rb1, Rb2, Rc, and Re. Extracts were found to contain $2.17 \mathrm{mg}, 1.51 \mathrm{mg}, 1.29 \mathrm{mg}$, and $0.46 \mathrm{mg}$ of ginsenoside $\mathrm{Rb} 1, \mathrm{Rb} 2, \mathrm{Rc}, \mathrm{Re}$, respectively, per gram weight. The ratios of each ginsenoside in the extracts were 1.0 : $0.7: 0.6: 0.2$, respectively. Taken together, the results indicate that HPLC-MS/MS spectrometry could be the most useful method for rapid analysis of even small amounts of major ginsenosides, while HPLC with UV detection could also be used for rapid analysis of major ginsenosides and for quality control of ginseng products.
\end{abstract}

Key words : Korean red ginseng extract, ginsenosides, HPLC-mass/mass, HPLC-UV

\section{INTRODUCTION}

Medicinal substances derived from plant extracts have traditionally been used for treating a number of common ailments. Their effects on the human body have been researched for a long time, especially in traditional oriental medicine. One important medicinal plant with a long medicinal history is ginseng (genus Panax, family Arialiaceae). There are six species of the Panax genus that are used medicinally, including Korean ginseng (Panax ginseng C.A. Mayer), American ginseng (Panax quinquefolium L.), Samchil (Panax notoginseng Burkill), Jukjeolsam

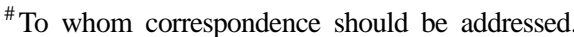

(Tel) +82-11-9115-7875; (Fax) +82-2-940-2861

(E-mail)dwlove@korea.ac.kr
}

(Panax japonicum C.A. Meyer), Samyeopsam (Panax trifoliusL.), and Himalayan ginseng (Panax pseudoginseng Wall.). Among these, Korean ginseng, a herbaceous perennial, is the most popular medicinal plant in Asia, including Korea, Japan, and China.

The pharmacological properties of ginseng are generally attributed to its triterpene glycosides, the ginsenosides. ${ }^{1)}$ A ginsenoside is a natural material that has been found to have various pharmacological actions helpful for human health. Ginsenosides have been used to treat and prevent atherosclerosis, asthma, diabetes, and endotoxin hepatotoxicity. ${ }^{2-4)}$ Ginsenosides are structurally described as glycosides of a triterpene aglycone moiety and their molecular configuration can vary depending on the nature of the complex oligosaccharides of the glycoside portions. 
Ginsenosides can be classified into two groups based on their aglycone moieties, which can be either 20 (S)-protopanaxadiol (ginsenosides Ra1, Rb1, Rb2, Rb3, Rc, and $\mathrm{Rd}$ ) or 20 (S)-protopanaxatriol (ginsenosidesRe, Rg1, Rg2, and $\mathrm{Rh} 1)^{5)}$ Among the 35 ginsenosides identified so far, six (Rb1, Rb2, Rc, Rd, Re and Rg1) account for $90 \%$ of the total. ${ }^{1,6)}$

Methods for ginsenoside analysis from Panax extracts have included colorimetry, ${ }^{7)}$ TLC, ${ }^{8)}$ GC, ${ }^{9,10)}$ HPLC, ${ }^{11-13)}$ ion chromatography, ${ }^{14)}$ and radioimmunoassay. ${ }^{15,16)}$ Among these, HPLC is the most widely used method as it generates quantitative analytical data for each ginsenoside. To analyze the properties of ginsenosides in ginseng extracts, HPLC-mass spectrometry (HPLC-MS) or HPLC-mass/ mass spectrometry (HPLC-MS/MS) detectors are becoming increasingly popular today. Because of the complicated matrixes of ginseng extracts, other HPLC detectors can also provide other, albeit somewhat limited, results. Saponin-based components lack chromophores but can be identified in the ultrashort UV wavelengths (203-210 $\mathrm{nm})^{17)}$ However, this places limitations on the selection of mobile phases and gradient experiments used in HPLC coupled to UV detection (HPLC-UV). For example, methanol has a UV cut-off point of $205 \mathrm{~nm}$, which means that refractive index (RI) or evaporative light scattering (ELSD) detection must be used when methanol is present in the mobile phase. Acetic acid also must be avoided as it also interferes with UV detection. Phosphoric acid has better sensitivity with UV detection, but cannot be used in an ELSD detector. When compared with HPLC-UV, mass or tandem mass spectrometry analyses have several advantages. A mass spectrometry detector is more sensitive than other detectors and also has a unique specificity because it detects molecular weights. In addition, it can detect a wide variety of materials, from inorganic materials to protein, and most importantly, can provide information on molecular structure through fragmentation ion analysis. HPLC-MS/MS is capable of detecting desired constituents using selected ion monitoring (SIM) and selected reaction monitoring (SRM) methods. ${ }^{18)}$ The unique specificity and sensitivity of HPLC-MS can make the analysis of samples containing various matrixes more convenient and time-efficient. It is a logical methodology to be applied to the analysis of ginseng in Panax species.

Korean ginseng has been recognized as being superior to foreign ginseng, and this has been attributed to the kinds and amounts of saponin constituents found in Korean ginseng. Therefore, it is important to develop an accurate and time-efficient method for use in the analysis of its ginsenosides and quality evaluation of ginseng extracts. There are many studies that have verified the pharmacological effects of ginseng, but we currently lack studies on rapid analysis of the ginsenosides. It generally takes more than 15 and upwards of 40 minutes for HPLCMS or HPLC-UV analysis, respectively, for a quality evaluation of the major saponin composition of a ginseng extract. The current study used HPLC-MS/MS and HPLCUV to determine the optimal conditions for rapid analysis of the major ginsenosides in Korean red ginseng extract. Chromatographic fingerprinting is found to be a simple and time-efficient method for testing and quality control of ginsenosides in ginseng products.

\section{MATERIALS AND METHODS}

\section{Reagents}

Ginsenoside Rb1, Rb2, Rc, Re, and Rg1 and Korean red ginseng extract derived from the root of Panax ginseng were acquired from KT\&G Central Research Institute. Other reagents were acquired from Sigma-Aldrich (USA). For HPLC mobile phases, J.T. Baker (USA) HPLC-grade water and acetonitrile were used. Ginsenoside standards were diluted with methanol.

\section{Analysis conditions and instrumentation}

For HPLC analysis, samples of Korean red ginseng extract were diluted with distilled water 5-fold. Three $\mathrm{mL}$ of this sample was loaded into OASIS (waters HLB) C18 cartridge column activated by $3 \mathrm{~mL} \mathrm{MeOH}$ and $3 \mathrm{~mL}$ distilled water and eluted by $4 \mathrm{~mL} \mathrm{MeOH}$. The eluate was concentrated to one third of its volume using nitrogen gas, then filtered through a syringe filter and injected onto the HPLC column.

In this study, the HPLC-MS/MS detector used was an Applied Biosystems API 2000 triple-quadrupole mass spectrometer equipped with a Turboionspray source and controlled by Analysis Data System (Version 14.2). The HPLC system consisted of a Perkin-Elmer 200 series micro pump system and autosampler. Product ions and precursor ions were scanned to acquire standard mass data, which were then used to analyze the ginsenosides in Korean red ginseng extract under the Multiple Reaction Monitoring (MRM) mode.

For the analysis of ginsenosides in the HPLC-MS/MS the HPLC mobile phase consisted of A) acetonitrile and B) water with $0.01 \%$ acetic acid. The mobile phase was filtered and ultrasonically-processed to remove gas bubbles before use. The gradient program of the mobile phase 
Table 1. Triple Quadrupole HPLC-MS/MS detector operation condition for analysis of Korean red ginseng extract

\begin{tabular}{cc}
\hline \hline Condition & \\
\hline Curtain Gas (CUR) & $20 \mathrm{psi}$ \\
Collision Gas (CAD) & $5 \mathrm{psi}$ \\
IonSpray Voltage (IS) & $5500 \mathrm{~V}$ \\
Temperature (TEM) & $350^{\circ} \mathrm{C}$ \\
Ion Source Gas 1 (GS1) & $30 \mathrm{psi}$ \\
Ion Source Gas 2 (GS2) & $70 \mathrm{psi}$ \\
Interface Heater (ihe) & on \\
Ion Source & Turbo Spray \\
\hline
\end{tabular}

was a linear gradient, from (10:90) to (70:30) over 7 min. The column was a Phenomenex Luna $5 \mu \mathrm{C} 18(50 \times 2.0$ $\mathrm{mm}$ ) reversed phase column and the flow rate of the mobile phase was set to $0.2 \mathrm{~mL} / \mathrm{min}$. $20 \mu \mathrm{L}$ of sample was injected for analysis. Standard concentrations of ginsenosides were $0.1,0.5,1.0,5.0$, or $10.0 \mathrm{ug} / \mathrm{mL}$. Table 1 . shows the standard conditions for the HPLC-MS/MS detector operation for analysis of Korean red ginseng extract.

The HPLC-UV detector was a UV-visible detector and mobile phase was delivered by two pumps. $10 \mu \mathrm{L}$ of sample was injected using an autosampler. Ginsenosides in Korean red ginseng extract were analyzed using Two software.

For the analysis of ginsenosides using HPLC-UV, the mobile phase constituents were A) acetonitrile and B) water with $0.001 \%$ formic acid. The mobile phase was filtered and ultrasonically-processed to remove gas bubbles before use. The gradient program a linear gradient from A-B (22:78) to (26:74) over 8 minutes then A-B (35:65) to $13 \mathrm{~min}$ followed by A-B (40:60) to $25 \mathrm{~min}$. The column was a ZORBAX SB-C18 $(5 \mu \mathrm{m} 4.5 \times 150 \mathrm{~mm}$, Agilent) and the flow rate of was set to $0.7 \mathrm{~mL} / \mathrm{min}$. The sample injection volume was $10 \mu \mathrm{L}$. The column temperature was set to $35^{\circ} \mathrm{C}$ and the wavelength of detector was set to $203 \mathrm{~nm}$. Standard concentrations of ginsenosides were $100,300,500$, or $1000 \mathrm{ug} / \mathrm{mL}$.

\section{RESULTS AND DISCUSSION}

Many research studies that have analyzed the ginsenosides from Korean red ginseng extracts have used HPLCUV detection. However, HPLC-MS has lower detection limits than HPLC-UV, is less affected by interfering substances, and can greatly reduce the processing time. Therefore, many researchers are interested in using HPLC-MS to analyze ginsenosides. Although it has these advantages, the HPLC-mass detector is not an easy piece of equip- ment for general laboratory usage, as it is costly and requires highly trained technicians for maintenance. This study applied both the HPLC-MS/MS detector and the HPLC-UV detector to develop comparable methods for analysis of ginsenosides $\mathrm{Rb} 1, \mathrm{Rb} 2, \mathrm{Rd}$, and $\mathrm{Rc}$ from Korean red ginseng extract within a short period of time.

In first experiment, rapid analysis of ginsenosides using the HPLC MS/MS was studied. The MS/MS detector condition was set to analyze the ginsenoside $\mathrm{Rb} 1, \mathrm{Rb} 2$, $\mathrm{Rd}$, and Rc following HPLC separation and the Q1 mass spectrum was used to detect standard ginsenosides. Mass data of the standards were acquired by direct injection analysis in the single-quadrupole mode in positive and negative modes. The positive ion mass spectra of the ginsenoside $\mathrm{Rb} 1, \mathrm{Rb} 2$, and $\mathrm{Rd}$ cations had the $[\mathrm{M}+\mathrm{H}]^{+}$and $[\mathrm{M}+\mathrm{Na}]^{+}$molecular weights. In the positive ion mode, ginsenoside $\mathrm{Rb} 1$ cation generated $[\mathrm{M}+\mathrm{Na}]^{+}$ions in $\mathrm{m} / \mathrm{z}$ $1131.8, \mathrm{Rb} 2$ cation in $\mathrm{m} / \mathrm{z} 1101.7$, and Re cation in 969.5. Ginsenosides $\mathrm{Rb} 1, \mathrm{Rb} 2$, and $\mathrm{Re}$ cations also generated $[\mathrm{M}+\mathrm{H}]^{+}$ions in $\mathrm{m} / \mathrm{z} 1109.9, \mathrm{~m} / \mathrm{z} 1079.7$, and $\mathrm{m} / \mathrm{z}$ 947.3, respectively. The positive ion mass spectra of ginsenoside Rc was not tried as its molecular weight was same as that of ginsenoside $\mathrm{Rb} 2$. The negative ion mass spectra of the ginsenoside $\mathrm{Rb} 1$ anion had the $[\mathrm{M}-\mathrm{H}]^{-}$and $[\mathrm{M}+\mathrm{Na}-\mathrm{H}]^{-}$ molecular weights. In the negative ion mode, ginsenoside $\mathrm{Rb} 1$ generated $[\mathrm{M}-\mathrm{H}]^{-}$in $\mathrm{m} / \mathrm{z} 1107.6$ and $[\mathrm{M}+\mathrm{Na}-\mathrm{H}]^{-}$in $\mathrm{m} / \mathrm{z}$ 1129.3. According to this study, there was less noise in the negative mode than in the positive mode when the Q1 quadrupole was used. It is therefore apparently more beneficial to use the ESI negative mode when analyzing ginsenoside Rb1 using Q1 quadrupole. However, when the triple quadrupole mass detector is used, the Q1 quadrupole is not the only tool used for the analysis. Instead, it is better to use the MRM mode, which deploys Q1, Q2, and Q3, to eliminate interruptions and to improve sensitivity. As many previous studies have generally conducted ginsenoside MRM analysis in the positive mode, this study also used the positive mode. According to Kim et $a l,{ }^{19)} \mathrm{m} / \mathrm{z} 677.3$ and 675.4 molecular ions were detected in the full scan spectrum under the Icariin Electrospray ionization (ESI) positive mode and ESI negative mode, but the $\mathrm{m} / \mathrm{z} 675.4$ ion in the ESI negative mode was more than twice as strong as molecular ions in the positive mode. This was probably because the negative molecular ion $[\mathrm{M}-\mathrm{H}]^{-}$is more freely formed than is the positive molecular ion $[\mathrm{M}+\mathrm{H}]^{+}$and it is more than twice as strong in its ionization in the mass detector.

A collision-induced dissociation (CID) experiment was conducted to examine the condition of fragmentation in 
the ginsenoside MRM analysis. The CID process has various names, including nozzle-skimmer fragmentation, sampling cone-voltage fragmentation, and high-orifice potential fragmentation. The glycosidic linkages, the core, and the attached sugars were analyzed from the CID of $[\mathrm{M}+\mathrm{Na}]^{+}$. The CID spectra was acquired from the ginsenoside Rb1's $[\mathrm{M}+\mathrm{Na}]^{+}$. Precursor ion at $\mathrm{m} / \mathrm{z} 1131.4$ and fragment ion at $\mathrm{m} / \mathrm{z} 789.4$ and $\mathrm{m} / \mathrm{z} 365.0$ were observed. The $\mathrm{m} / \mathrm{z} 365.0$ ion is [Glucose - Glucose $+\mathrm{Na}]^{+}$generated from the decomposition of the glycosidic bond, while the $\mathrm{m} / \mathrm{z} 789.4$ ion is the rest of ginsenoside Rb1. Among the peaks mentioned above, the $\mathrm{m} / \mathrm{z} 365.0$ ion showed the strongest peak response.

Strong fragment ions were selected based on the CID experiment of ginsenoside $\mathrm{Rb} 1, \mathrm{Rb} 2, \mathrm{Rc}$, and $\mathrm{Re}$ and were used in a further MRM quantification experiment. For precursor ions of ginsenosides $\mathrm{Rb} 1, \mathrm{Rb} 2, \mathrm{Rc}$, and $\mathrm{Re}$, $\mathrm{m} / \mathrm{z}$ 1131, 1101, 1101, and 969 were chosen, respectively, and $\mathrm{m} / \mathrm{z} 365,335,335$, and 789 were used for fragment ions, respectively. As sensitivity was strongest in the MRM mode than in the scan mode in the triple quadrupole mass detector, this study used the MRM mode to quantify ginsenosides Rb1, Rb2, Rc, and Re. According to Kim et al., ${ }^{19)}$ the optimization of MRM conditions involves selecting more than three fragment ions from one molecular ion and then choosing two fragment ions with less matrix effect and interference, to conserve the equipment parameters required for the generation of fragment ions. In the MRM mode, molecular ions are selected in tandem mass Q1 and energy is applied to these molecular ions in Q2 to cause them to fragment. In Q3, then, fragment ions are selected to identify the constituents and allow quantification. ${ }^{20)}$

The standard ginsenosides Rb1, Rb2, Rc, Re were analyzed with HPLC-triple quadrupole mass detection in the positive MRM mode. For a mobile phase, acetonitrile and water with $0.01 \%$ acetic acid were used and a gradient program was generated to modify the acetonitrile percentage from $10 \%$ to $70 \%$ over seven minutes. Fig. 1 shows the chromatogram of standard ginsenosides $\mathrm{Rb} 1, \mathrm{Rb} 2, \mathrm{Rc}$, and $\mathrm{Re}$. The retention order was Re, Rb1, Rc, and $\mathrm{Rb} 2$ and the retention times were 3.3, 5.4, 5.6, and $5.8 \mathrm{~min}$, respectively. All of the ginsenoside peaks were separated within seven minutes.

Fig. 2 shows the chromatogram of the ginsenosides $\mathrm{Rb} 1, \mathrm{Rb} 2, \mathrm{Rc}$, and Re from Korean red ginseng extract. The positive MRM mode was applied for the separation of each constituent. Then, the precursor ion, fragment ion and retention time of each ginsenoside constituent were compared to those of standard ginsenosides. The separation order and retention time for each constituent were similar to those of standard ginsenosides. The peaks for $\mathrm{Rb} 1, \mathrm{Rb} 2$, and $\mathrm{Rc}$ were high, but the Re peak was relatively low. Without interference from other constituents in the sample or matrix, all of the Rb1, Rb2, Rc, and Re peaks from Korean red ginseng extract were separated within seven minutes. $1 \mathrm{~g}$ of Korean red ginseng extract contained $2.17 \mathrm{mg}$ ginsenoside $\mathrm{Rb} 1,1.51 \mathrm{mg}$ ginsenoside $\mathrm{Rb} 2,1.29 \mathrm{mg}$ ginsenoside $\mathrm{Rc}$, and $0.46 \mathrm{mg}$ ginsenoside

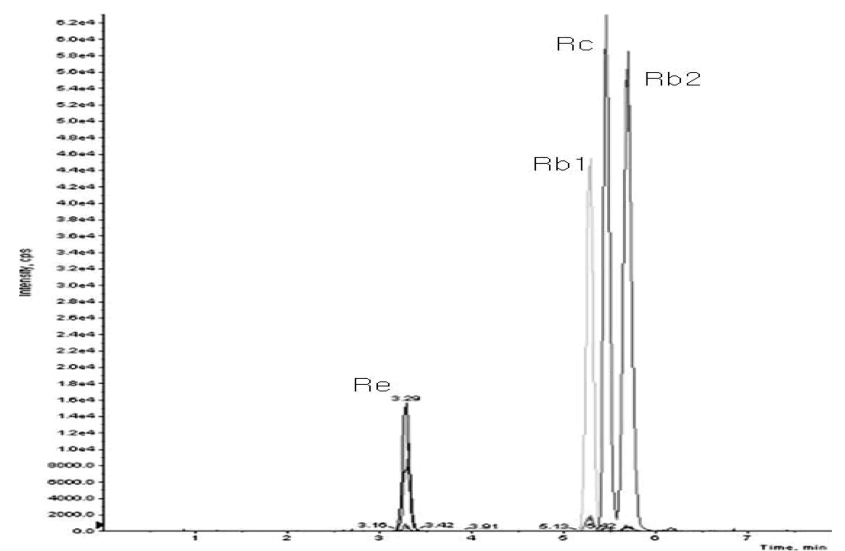

Fig. 1. Chromatogram of ginsenoside $\mathrm{Rb} 1, \mathrm{Rb} 2, \mathrm{Rc}$, and $\mathrm{Re}$ Standard in HPLC-MS/MS analysis. The retention order was ginsenoside $\mathrm{Re}, \mathrm{Rb} 1, \mathrm{Rc}$, and $\mathrm{Rb} 2$ and the retention times were 3.3, 5.4, 5.6, and $5.8 \mathrm{~min}$, respectively. (Phenomenex Luna 5 u C18 $(50 \times 2.0 \mathrm{~mm})$, linear gradient with acetonitrile and water, $0.2 \mathrm{~mL} / \mathrm{min}$ ).

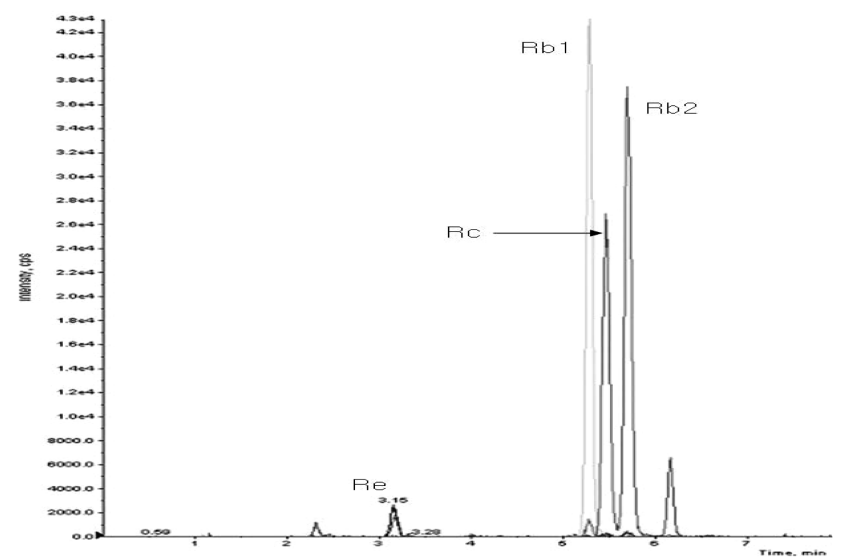

Fig. 2. Chromatogram of ginsenoside $\mathrm{Rb} 1, \mathrm{Rb} 2$, Rc, and $\mathrm{Re}$ in Korean red ginseng extract in HPLC-MS/MS analysis. The retention order was ginsenoside $\mathrm{Re}, \mathrm{Rb} 1, \mathrm{Rc}$, and $\mathrm{Rb} 2$ and the retention times were 3.2, 5.3, 5.5, and $5.7 \mathrm{~min}$, respectively. (Phenomenex Luna 5 u C18 $(50 \times 2.0 \mathrm{~mm})$, linear gradient with acetonitrile and water, $0.2 \mathrm{~mL} / \mathrm{min}$ ). 
Rc. The relative ratios of ginsenosides $\mathrm{Rb} 1, \mathrm{Rb} 2, \mathrm{Rc}$, and $\mathrm{Re}$ in Korean red ginseng extract were $1.0: 0.7: 0.6: 0.2$. Korean red ginseng extract contained the highest content of ginsenoside $\mathrm{Rb} 1$, followed by $\mathrm{Rb} 2, \mathrm{Rc}$, and Re. The chromatograms indicate a stable baseline with stable separation without any interference due to high mass/mass selectivity and with a clearly shaped peak. The detection limits for ginsenosides Rb1, Rb2, Rc, and Re were 18, 16, 14 , and $23 \mathrm{ng} / \mathrm{mL}$, respectively. This retention time of seven minutes is shortest reported to date among the studies of major ginsenoside analysis in literature. This HPLCmass/mass method could be used for rapid measurement of concentration and chromatographic fingerprinting for quality evaluation of major ginsenosides in ginseng extract.

In the second experiment, the HPLC-UV system was used to analyze standard ginsenosides $\mathrm{Rb} 1, \mathrm{Rb} 2, \mathrm{Rc}$, and Re. For a mobile phase, acetonitrile and water with $0.001 \%$ formic acid were used and a gradient program was generated to modify the acetonitrile percentage from $22 \%$ to $40 \%$ in 25 minutes. The order of separation was ginsenoside Re, Rb1, Rc, and Rb2, which was the same as seen with the HPLC-MS/MS system. However, the total retention time was prolonged up to 25 minutes. The retention time for ginsenosides Re, Rb1, Rc, and Rb2 were 6.5, 20.5, 21.7, and $22.6 \mathrm{~min}$, respectively. Each peak was clearly separated in the chromatogram without no interference from other peaks. If the conditions of mobile phase were set to isocratic using acetonitrile and water, the peaks were not easily separated and the standard detection limit was high. Fig. 3 shows a chromatogram of a typical analysis of ginsenosides Rb1, Rb2, Rc, and Re from Korean red ginseng extract using HPLC-UV. Under the conditions used this study, ginsenosides Re, Rb1, Rc, and $\mathrm{Rb} 2$ in Korean red ginseng extract were easily separated in 25 minutes. The chromatogram was not as clear as the results obtained from the mass detector, but the separation was stable without any major intereferences. In this study, the detection limits for ginsenosides Rb1, Rb2, Rc, and Re were $14,13,16$, and $21 \mu \mathrm{g} / \mathrm{mL}$, respectively.

Most previous studies on the analysis of ginsenosides in ginseng have used UV, ELSD, or RI detectors connected to an HPLC system. As these methods could not support quantification when there were interferences and peak overlaps, the conditions of the mobile phase had to be controlled to maintain maximum separation for quantification of complicated mixtures. Consequently, each sample generally required at least 40 minutes for separation to ensure accuracy and precision. Thus, the previously used HPLC-RI, HPLC-UV, and HPLC-ELSD methods were

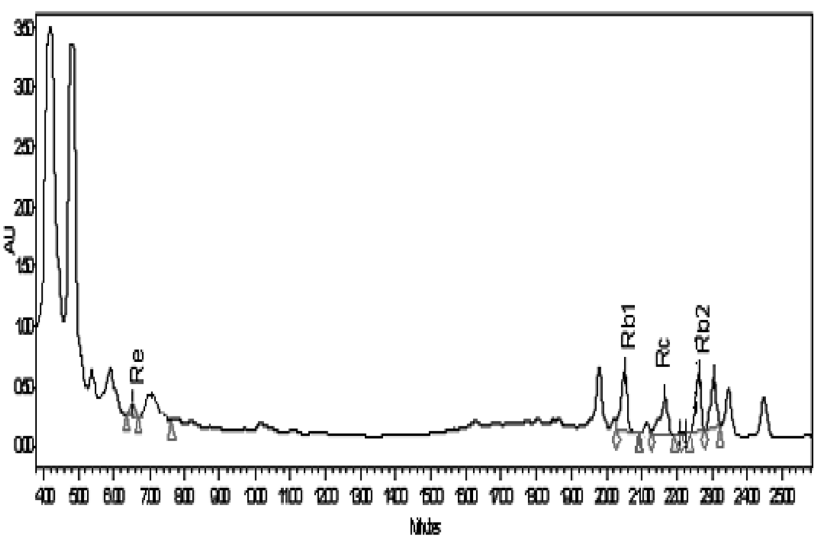

Fig. 3. Chromatogram of ginsenoside $\mathrm{Rb} 1, \mathrm{Rb} 2, \mathrm{Rc}$, and $\mathrm{Re}$ in Korean red ginseng extract in HPLC-UV analysis. The retention order was ginsenoside $\mathrm{Re}, \mathrm{Rb} 1, \mathrm{Rc}$, and $\mathrm{Rb} 2$ and the retention times were $6.5,20.5,21.7$, and $22.6 \mathrm{~min}$, respectively. (ZOBAX SB-C18 (5 um $4.5 \times 150 \mathrm{~mm}$ ), linear gradient with acetonitrile and water, $0.7 \mathrm{~mL} / \mathrm{min}$ ).

limited by selectivity as well as by their long time for analysis. To overcome these limitations, HPLC-MS or HPLC-MS/MS (triple quadrupole or ion trap) systems have been more intensively investigated in recent studies. An HPLC-MS system generally uses a HPLC-MS/MS tandem detection system. Tandem HPLC-MS/MS is more widely used because it can remove interferences more effectively than can simple HPLC-MS. Triple quadrupole HPLC-MS/MS is better for the quantification studies than is ion trap HPLC-MS/MS. The ion trap HPLC-MS/MS is more suitable for structural analysis of compounds. Ligor et al., ${ }^{21)}$ achieved a $30 \mathrm{ng} / \mathrm{mL}$ or lower detection limit when they analyzed ginsenosides using ion trap LC-MS/ MS. In the current study, HPLC-MS/MS had a standard detection range of $0.1-10 \mu \mathrm{g} / \mathrm{mL}$ and the detection limit was 14-23 ng/mL. The detection range for HPLC-UV standard was $100-1000 \mu \mathrm{g} / \mathrm{mL}$ and the corresponding detection limit was $13-21 \mu \mathrm{g} / \mathrm{mL}$. This indicated that the detection sensitivity of HPLC-MS/MS was greater than that of HPLC-UV.

Co-elution of ginsenosides $\mathrm{Rg} 1$ and $\mathrm{Re}$ is a common problem that has been previously reported. ${ }^{22,23)}$ The separation of $\mathrm{Re}$ and $\mathrm{Rg} 1$ can be achieved with a mobile phase consisting of no more than $20 \%$ acetonitrile in water. ${ }^{24)}$ Therefore, acetonitrile has to be maintained below $20 \%$ for up to nine minutes, to allow elution of separated ginsenosides $\operatorname{Re}$ and $\operatorname{Rg} 1$. The downside of this separation is that it adds a great deal of time to each separation. In the current study using UV detector analysis, this condition was neglected in favor of fast analysis. Therefore, $\operatorname{Rg} 1$ 
could not be analyzed using the HPLC-UV analysis conditions. On the other hand, $\operatorname{Rg} 1$ was easily analyzed using HPLC-MS/MS. The retention time of $\mathrm{Rg} 1$ and Re were 3.2 and 3.3 min, respectively, which are very similar peak retention times, but it was not difficult to analyze them as their molecular weights are 801 and 947, respectively.

Today, many kinds of ginseng products are being imported, exported and circulated in the market. For quality evaluation, identification, and storage stability tests of these products, it is necessary to develop a simple and fast analysis method for the ginsenoside content of ginseng products. This study shows that a simple and fast HPLCUV method can be utilized as a general quality control method and can be applied to chromatographic fingerprinting of Panax ginseng products. This rapid HPLC-UV fingerprinting method is based on the different contents of four major ginsenosides for the quality evaluation of Panax ginseng products and is better than a pharmacopoeia, in which only ginsenoside Rb1 is used as the marker of Panax quinquefolium $L^{25}$ ) The HPLC-MS or the ultra-performance liquid chromatograph system is faster and more accurate, but any HPLC-UV instrument can be used for the general quality control of ginseng products or extracts when there is no such expensive instrument available.

This study found that $1 \mathrm{~g}$ of Korean red ginseng extract contains $2.17 \mathrm{mg}$ ginsenoside $\mathrm{Rb} 1,1.51 \mathrm{mg}$ ginsenoside $\mathrm{Rb} 2,1.29 \mathrm{mg}$ ginsenoside $\mathrm{Rc}$, and $0.46 \mathrm{mg}$ ginsenoside Rc. This means that there are $0.22,0.15,0.13$, and $0.05 \%$ of ginsenoside $\mathrm{Rb} 1, \mathrm{Rb} 2, \mathrm{Rc}$, and Re, respectively. The relative ratio of ginsenoside $\mathrm{Rb} 1, \mathrm{Rb} 2, \mathrm{Rc}$, and $\mathrm{Re}$ in Korean red ginseng extract was $1.0: 0.7: 0.6: 0.2$, respectively. According to Choi $e t a l,{ }^{26)}$ there were $0.32,0.21$, 0.18 , and $0.08 \%$ of ginsenosides $\mathrm{Rb} 1, \mathrm{Rb} 2, \mathrm{Rc}$, and $\mathrm{Re}$, respectively, in Korean red ginseng extract, which was similar to the results of this study. Another study on main root Panax ginseng showed 0.18-0.27, 0.17-0.25, 0.430.52 , and $0.30-0.37 \%$ of ginsenosides Rb1, Rb2, Rc, and $\mathrm{Re}$, respectively. ${ }^{27)}$ Another study on red, white and extruded root Panax ginseng concluded that there are $0.86-1.43,0.53-1.18,0.52-1.09$, and $0.96-1.74 \mathrm{mg} / \mathrm{g}$ of ginsenoside $\mathrm{Rb} 1, \mathrm{Rb} 2, \mathrm{Rc}$, and Re, respectively. ${ }^{28)}$ These results were similar to the results of this study. Another study on Panax ginseng extracts showed 7.91, 7.23, 8.23. $5.25 \mathrm{mg} / \mathrm{g}$ of ginsenoside Rb1, Rb2, Rc, and Re, but their relative ratios were similar to the results of this study. Most previous analyses on the contents of Korean ginseng showed results that corresponded to the results of the current study. Therefore, the HPLC-UV method reported here can be readily applied to measure the ratio of major ginsenosides and quickly evaluate the quality of ginseng products.

This study showed that HPLC-MS/MS could be the most useful method for rapid analysis of even small amounts of major ginsenosides, but that HPLC-UV could also be used for rapid analysis of major ginsenosides in ginseng products and for quality control of ginseng.

\section{ACKNOWLEDGEMENT}

This work was supported by the grant from the Korean Society of Ginseng.

\section{REFERENCES}

1. Tyler, V. : Rational Phytotherapy 4th ed. Springer. Berlin, Germany (1999).

2. Kim, H. S., Hong, Y. T. and Jang, C. G. : Effects of the ginsenosides $\mathrm{Rg} 1$ and $\mathrm{Rb} 1$ on morphine-induced hyperactivity and reinforcement in mice. J. Pharm. Pharmacol. 50, 555560 (1998).

3. Kim, Y. C., Kim, S. R., Markelonis, G. J. and Oh, T. H. : Ginsenosides $\mathrm{Rb} 1$ and $\mathrm{Rg} 3$ protect cultured rat cortical cells from glutamate-induced neurodegeneration. J. Neurosci. Res. 53, 426-432 (1998).

4. Yokozawa, T., Liu, Z. W. and Dong, E. : A study of ginsenoside-Rd in a renal ischemia-reperfusion model. Nephron Basel, 78, 201-206 (1998).

5. Gillis, C. N. : Panax ginseng pharmacology: A nitric oxide link?. Biochem. Pharmacol. 54, 1-8 (1997).

6. Li, W., Gu, C., Zhang, H., Awang, D. V. C., Fitzloff, J. F., Fong, H. H. S. and van Breeman, R. B. : Use of high performance liquid chromatography-tandem mass spectrometry to distinguish Panax ginseng C.A. Meyer (Asia ginseng) and Panax quinquefolius L. (North American ginseng). Anal. Chem. 72, 5417-5422 (2000).

7. Woo, L. K., Han, H. H., Baik, D. W. and Park, D. S. : Characterization of ginseng extracts. J. Pharm. Soc. Kor. 17, 129 (1973).

8. Sanada, S., Shibata, S. and Shoji, J. : Quantitative analysis of ginseng saponins. Yakugaku Zasshi. 98, 1048-54 (1978).

9. Bombardelli, E., Bonat, A., Gabetta, B. and Martinelli, E. M. : Gas-liquid chromatographic method for determination of ginsenosides in Panax ginseng. J. Chromatogr. 196, 121132 (1980).

10. Park, M. K., Park, J. I., Kang, J. S., Lee, M. Y., Park, Y. I., Yu, S. J. and Han, B. H. : Rapid hydrolysis of ginseng saponin by microwave oven reaction. Kor. J. Ginseng Sci. 17, 3538 (1993).

11. Ko, S. R., Choi, K. J., Kim, S. C. and Kim, M. W. : Original 
articles; Contents of crude saponin and ginsenosides in white ginsengs. Kor. J. Pharmacogn. 20, 170-174 (1989).

12. Soldati, F. and Sticher, O. : HPLC separation and quantitative determination of ginsenosides from Panax ginseng, Panax quinquefolium and from ginseng drug preparations. 2nd communication. Planta Medica. 38, 348-357 (1980).

13. Park, M. K. : Analysis of ginsenosides by thermospray LC/ MS. Korean J. Ginseng Sci. 19, 134-137 (1995).

14. Park, M. K., Park, J. H., Lee, M. Y., Kim, S. J. and Park, I. J. : Microanalysis of ginseng saponins by ionchromatography with pussed amperometric detection. J. Liq. Chromatogr. 17, 1171 (1994).

15. Han, B. H. and Han, Y. N. : Immunochemical assay for Korean ginseng saponins I synthesis of ginsenoside - protein conjugate. J. Pharm. Soc. Kor. 25, 43-47 (1981).

16. Sankawa, U., Shibuya, M., Ebizuka, Y., Noguchi, H., Kinoshita T., Iitaka, Y., Endo, A. and Kitahara, N. : Depside as potent inhibitor of prostaglandin biosynthesis: a new active site model for fatty acid cyclooxygenase. Prostaglandins. 24, 2134 (1982)

17. Wan, J. B., Yang, F. Q., Li, S. P., Wang, Y. T. and Cui, X. M. : Chemical characteristics for different parts of Panax notoginseng using pressurized liquid extraction and HPLC-ELSD. J. Pharm. Biomed. Anal. 41, 1596-1601 (2006).

18. Park, J. H. : LC/MS Interface. Pharmacon, 21, 30 (1991).

19. Kim, H. Y., Lee, H. M., Jang, Y. M., Joo. H. J., Jung, Y. H., Lee, M. S., Park, J. S. and Lee, K. H. : Development of rapid analytical metod of forbidden medicines in dietary supplements using LC-ESI-Tandem MS. Korean J. Food Sci. Technol. 39, 372-379 (2007).

20. Koesukwiwat, U., Jayanta, S. and Leepipatpiboon, N. : Validation of a liquid chromatography-mass spectrometry multiresidue method for the simultaneous determination of sul- fonamides, tetracyclines and pyrimethamine in milk. $J$. Chromatogr. A. 1140, 147-156 (2007).

21. Ligor, T., Ludwiczuk, A., Wolski, T. and Buszewski, B. : Isolation and determination of ginsenosides in American ginseng leaves and root extracts by LC-MS. Anal. Bioanal. Chem. 383, 1098-1105 (2005).

22. Bonfill, M., Casals, I., Palazon, J., Mallol, A. and Morales, C. : Improved high performance liquid chromatographic determination of ginsenosides in Panax ginseng-based pharmaceuticals using a diol column. Biomed. Chromatogr. 16, 68-72 (2002).

23. Lee, H. K., Koh, H. L., Ong, E. S. and Woo, S. O. : Determination of ginsenosides in medicinal plants and health supplements by pressurized liquid extraction (PLE) with reversed phase high performance liquid chromatography. $J$. Sep. Sci. 25, 160-166 (2002).

24. Ma, X. Q., Wang, L. X., Xu, Q., Zhang, F., Xiao, H. B. and Liang, X. M. : Optimization method of multi-segment gradient for separation of ginsenosides in reversed phase-high performance liquid chromatography. Chem. J. Chin. Univ. 25, 238-242 (2004).

25. Chinese pharmacopoeia. 1, 99-100 (2000).

26. Choi, K. J., Ko, S. R., Kim, S. C. and Park, J. D. : Saponin and ginsenoside content in Korean red ginseng products. Korean J. Ginseng Sci. 13, 178-182 (1989).

27. Ha, D. C., Lee, J. W., Do, J. H., Park, C. K. and Ryu, G. H. : Drying rate and physicochemical characteristic of dried ginseng root at different temperature. J. Korean Soc. Food Sci. Nutr. 33, 741-746 (2004).

28. Ha, D. C. and Ryu, G. H. : Chemical components of red, white and extruded root ginseng. J. Korean Soc. Food Nutr. 34, 247-254. (2005). 